\title{
Comparison of Packed Cell Volume Changes after Erythropoietin Administration and Blood Transfusion in Patients with Anemia of Chronic Kidney Disease on Hemodialysis
}

\author{
Anju Bajracharya, ${ }^{1}$ Jayendra Bajracharya ${ }^{2}$ \\ ${ }^{1}$ Department of Pharmacy, Madan Bhandari Academy of Health Sciences, Hetauda, Makawanpur, Nepal; \\ ${ }^{2}$ Department of Lab Medicine, Madan Bhandari Academy of Health Sciences, Hetauda, Makawanpur, Nepal.
}

\begin{abstract}
Introduction: Anemia of chronic kidney disease (CKD) can be managed by regular administration of erythropoiesis stimulating agents (ESAs) and/or blood transfusion. The response to these therapies can be monitored by serial packed cell volume (PCV).

Objective: This study was done to compare the temporal changes in PCV after ESA therapy and blood transfusion in patients with recently diagnosed anemia in CKD stage 5 on hemodialysis (CKD 5 HD).

Methods: Medical records of patients undergoing hemodialysis at the National Kidney Center, Balaju, Kathmandu from July to September 2013 were examined retrospectively. The data collected were analyzed using Minitab 16.

Results: A total of 44 patients were on ESA therapy while 48 patients were on blood transfusion. The mean PCV at the start of blood transfusion was significantly lower than the mean PCV at the start of ESA therapy $(p=0.000)$ but at four weeks, the mean PCV in blood transfusion group was significantly greater than that in ESA therapy group $(p=0.008)$. At eight weeks and twelve weeks, the mean PCVs in both groups were not significantly different from each other $(\mathrm{p}=0.949$ and $\mathrm{p}=0.747)$.

Conclusions: Blood transfusion increases PCV immediately and in sustained manner while with ESA therapy, the response takes a longer time to manifest. A large number of non-responders to ESA therapy may have influenced the findings of this study. It is recommended that adequate dosing of ESA and attention to comorbid conditions be followed during ESA therapy.

Keywords: Anemia; chronic renal insufficiency; blood transfusion; hematinics.
\end{abstract}

\section{INTRODUCTION}

The anemia of chronic kidney disease (CKD) is primarily caused by deficiency of erythropoietin as the erythropoietin-producing interstitial fibroblastic cells are damaged. The deterioration of CKD, marked by a progressive decline in glomerular filtration rate (GFR) is associated with declines in hematological parameters, such as PCV or hemoglobin level and GFR in CKD. ${ }^{1}$

\section{Correspondence}

Anju Bajracharya

Email:anjubajra@gmail.com

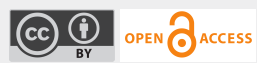

Citation

Bajracharya A, Bajracharya J. Comparison of Packed Cell Volume Changes after Erythropoietin Administration and Blood Transfusion in Patients with Anemia of Chronic Kidney Disease on Hemodialysis. Nepal J Health Sci. 2021 Jul-Dec; 1(2):48-51.
CKD Stage 5 is the most severe stage of CKD, requiring regular hemodialysis (CKD $5 \mathrm{HD}$ ). These patients are severely anemic and require blood transfusions and/or erythropoiesis stimulating agents (ESAs) such as human recombinant erythropoietin (Epoetin and Darbepoetin) for improvement of their hematological parameters. ${ }^{2}$

National Kidney Center, Balaju, Kathmandu implemented the best practice target of PCV of 30 for patients with CKD 5 HD undergoing ESA therapy or blood transfusion. Variation in hematological parameters in CKD $5 \mathrm{HD}$ patients undergoing these therapies is related to adjustments in ESA therapy or blood transfusion, variation in hydration status, inflammatory processes, correction of iron deficiency, blood loss such as due to access surgery and dialysis adequacy. ${ }^{3}$ 
This study aimed to compare the changes in PCV between patients on ESA therapy and blood transfusion at the start, four weeks, eight weeks, and twelve weeks of therapies.

\section{METHODS}

This study is a retrospective study involving collection of secondary data from the medical records of the CKD 5HD patients undergoing hemodialysis and receiving either an ESA agent or blood transfusion from July 2013 to September 2013 (12 weeks) at National Kidney Center, Balaju, Kathmandu. Ethical permission was obtained from the authorities at the National Kidney Center for collection of secondary data in a retrospective manner. As this was a durationbased study (12 weeks), sample size calculation was not done and all patients visiting the National Kidney Center for hemodialysis during the study period were included in this study. Anonymization of identifying details of the patients was done by assigning a code number for each patient. Patients were selected according to the predefined inclusion and exclusion criteria. Patients recently diagnosed with CKD 5 on regular hemodialysis, and on either ESA therapy or blood transfusion were included in the study. Patients on both ESA therapy and blood transfusion were excluded. Data collection forms were filled from the medical records of the patients in August 2013. Minitab 16 was used to perform the required statistical tests.

\section{RESULTS}

A total of 107 patients were included in this study, with 44 patients on ESA therapy alone and 48 patients on blood transfusion alone and the remaining 15 patients were switched from erythropoietin therapy to blood transfusion or vice versa and thus, were excluded.

\section{ESA Therapy Group}

For the patients receiving an ESA alone, the mean baseline PCV (right before receiving the first dose of erythropoietin) was 26.55. The mean PCV at four weeks of ESA therapy was 26.79 and the $95 \%$ confidence limits for the mean PCV were 25.38 and 28.19. The mean PCV at eight weeks of ESA therapy was 28 and the $95 \%$ confidence limits for the mean PCV were 26.70 and 29.29. The mean PCV at twelve weeks of ESA therapy was 28 and the $95 \%$ confidence limits for the mean PCV were 26.34 and 29.65. Comparing all the mean PCVs at the start and at four weeks, eight weeks and twelve weeks of ESA therapy, no significant difference was observed among the mean PCVs $(p=0.463$, One way ANOVA). With the given statistical results, it is concluded that the ESA administration did not bring any significant change in the PCV level even after twelve weeks of ESA administration.

\section{Blood Transfusion Group}

For the CKD $5 \mathrm{HD}$ patients receiving blood transfusion alone, the mean baseline PCV (right before the start of blood transfusion) was 23.48. The mean PCV at four weeks of intermittent blood transfusion was 29.37 and the $95 \%$ confidence limits for the mean PCV were 28.07 and 30.67. The mean PCV at eight weeks of intermittent blood transfusion was 28.31 and the $95 \%$ confidence limits for the mean PCV were 26.99 and 29.62. The mean PCV at twelve weeks of intermittent blood transfusion was 28.31 and the $95 \%$ confidence limits for the mean PCV were 27.26 and 29.35. Comparing all the mean PCVs at the start and at four, eight, and twelve weeks of intermittent blood transfusion, the mean PCVs were found to be significantly different ( $p=0.000$, One way ANOVA).

Comparison of PCVs of patients undergoing ESA therapy and blood transfusion

The mean PCV at the start of blood transfusion was significantly lower than the mean PCV at the start of ESA therapy ( $p=0.000$, Pooled t-test). The mean PCV at four weeks of intermittent blood transfusion was also significantly greater than the mean PCV at 
four weeks of ESA therapy ( $p=0.008$, Pooled t-test). The mean PCV at eight weeks of intermittent blood transfusion and the mean PCV at eight weeks of ESA therapy were not significantly different ( $p=0.949$, Pooled t-test). The mean PCV at twelve weeks of intermittent blood transfusion and the mean PCV at twelve weeks of ESA therapy were not significantly different ( $p=0.747$, Pooled t-test).

\section{DISCUSSION}

Even though the mean baseline PCV is significantly lower in patients on blood transfusion as compared to patients on ESA therapy, the mean PCVs are quite different after the therapies. Mean PCV increased significantly after 4 weeks of blood transfusion, however, this increment was not seen with ESA therapy confirming that blood transfusion increases PCV immediately and in sustained manner while with ESA therapy, the response takes a longer time to manifest (at least 8 weeks in this study). In different literatures, it has been claimed that the ESA therapy increases PCV usually within two to six weeks reaching the best practice target level of PCV of 30 within eight weeks. Surprisingly, the mean PCVs at the start and four, eight, and twelve weeks of ESA therapy were not significantly different; this clinically translates to ineffective ESA therapy in these patients. In the post-hoc analysis of 44 patients on ESA therapy, only 18 patients were found to reach the best practice target PCV level of 30 by the end of this study period, with an average of 7.29 weeks of ESA therapy. This discrepancy in PCV levels in patients on ESA therapy could be due to multiple reasons, the major reason being the dose of an ESA - inadequate initial dose and/or subsequent adjusted dose.

According to the National Kidney Foundation Kidney Disease: Improving Global Outcomes (KDIGO) Clinical Practice Guideline, adequate initial dose of epoetin-alfa or epoetin-beta is between 20 to $50 \mathrm{IU} / \mathrm{kg}$ body weight with a dosing frequency of three times a week. Epoetin-alfa or epoetin-beta dosage may subsequently be increased every four weeks by a weekly dose of $60 \mathrm{IU} / \mathrm{Kg}$ if the increase in hematological parameters is not adequate. If adequate doses of an ESA are not administered, the incremental changes in PCV will not be observed. Additionally, the effect of an adequate dose of an ESA takes longer time to manifest.

The second reason can be the poor response to the ESA therapy, which may result from history of cardiovascular disease, iron deficiency, inflammatory conditions and obesity. ${ }^{4}$ Moreover, adequate dialysis is almost always a prerequisite for correction of anemia and the progressive increments seen in PCV and/or hemoglobin. ${ }^{5}$ Examination of these factors were not in the scope of this study.

While blood transfusion seems to have a favorable effect on PCV in patients with CKD $5 \mathrm{HD}$, it must be recognized that blood transfusion carries multiple life-threatening risks, such as transfusion reactions and blood-borne infections, which will compound the multitude of problems patients with CKD 5 HD are already facing. ${ }^{6}$

A limited sample size in regards to the number of patients available for the ESA therapy arm and for the blood transfusion arm is one of the limitations of this study.

\section{CONCLUSIONS}

Response to ESA therapy in CKD $5 \mathrm{HD}$ patients depends on dose adequacy and presence of other comorbid factors. If dose adequacy and other comorbid factors are not accounted for, the response to ESA therapy may not be seen even up to 12 weeks of therapy. It is thus recommended that these factors be considered at the beginning of ESA therapy. It is also equally important to see the response to ESA therapy frequently and adjust the dose accordingly. As a future exploration, all factors that influence response to ESA therapy should be studied. 
It is without doubt that blood transfusion would show a much instantaneous impact on PCV in CKD 5 HD as it simply brings in massive numbers of donor RBCs into the circulation of the patient. Owing to high risks of transfusion reactions and blood-borne infections, blood transfusion must be considered the last resort to address anemia in patients with CKD 5 HD.

\section{ACKNOWLEDGEMENT}

We would like to acknowledge the National Kidney Center, Balaju, Kathmandu, Nepal for permitting to conduct this study.

\section{Conflict of Interest: None}

\section{REFERENCES}

1. Lerma EV, Nissenson AR, editors. Nephrology secrets. Third edition. Philadelphia, Pa: Elsevier, Mosby; 2012. 647 p.

2. Shahab MH, Saifullah Khan S. Erythropoietin Administration for Anemia Due to Chronic Kidney Disease - Subcutaneous OR Intravenous, What Do We Know So Far? Cureus. 2020 Sep 10;12(9):e10358. doi: 10.7759/cureus.10358. PMID: 33062481; PMCID: PMC7549864.

3. Santos EJF, Dias RSC, Lima JFB, Salgado Filho N, Miranda dos Santos A. Erythropoietin Resistance in Patients with Chronic Kidney Disease: Current Perspectives. Int J Nephrol Renovasc Dis. 2020;13:231-237 https://doi.org/10.2147/IJNRD.S239151

4. Mehdi U, Toto RD. Anemia, diabetes, and chronic kidney disease. Diabetes Care. 2009 Jul;32(7):1320-6. doi: 10.2337/dc08-0779. PMID: 19564475; PMCID: PMC2699743.

5. Rosati A, Ravaglia F, Panichi V: Improving Erythropoiesis Stimulating Agent Hyporesponsiveness in Hemodialysis Patients: The Role of Hepcidin and Hemodiafiltration Online. Blood Purif 2018;45:139-146. doi: 10.1159/000485314

6. Tanhehco YC, Berns JS. Red blood cell transfusion risks in patients with end-stage renal disease. Semin Dial. 2012 Sep-Oct;25(5):539-44. doi: 10.1111/j.1525139X.2012.01089.x. Epub 2012 Jun 11. PMID: 22686519; PMCID: PMC3676886. 